\title{
ARTICLE
}

\section{Development of dc and ac magnetic-measurement system for a ferromagnetic superconductor, uranium digermanide}

\author{
Akira Yamaguchi $^{\mathrm{a}^{*}}$, Hiroyuki Tanaka ${ }^{\mathrm{a}}$, Ikuto Kawasaki ${ }^{\mathrm{a}}$, Akihiko Sumiyama ${ }^{\mathrm{a}}$, \\ Gaku Motoyama ${ }^{\mathrm{b}}$ and Tomoo Yamamura ${ }^{\mathrm{c}}$ \\ ${ }^{a}$ Graduate School of Material Sciences, University of Hyogo, 3-2-1 Kouto, Kamigoricho, Ako-gun, Hyogo, 678-1297, Japan; \\ ${ }^{b}$ Department of Physics and Materials Science, Shimane University, Matsue, Shimane, 690-8504, Japan; ${ }^{c}$ Institute for Materials \\ Research, Tohoku University, 2-1-1 Katahira, Aoba-ku, Sendai, Miyagi, 980-8577, Japan
}

\begin{abstract}
Development of a magnetic measurement system for investigation of ferromagnetic-superconducting phase of uranium compounds is reported. The system consists of two kinds of magnetic sensors: One is a Hall-effect sensor which is used to measure large ferromagnetic signal from the sample, and the other is superconducting-quantum-interference-device (SQUID) sensor, which is used to detect much smaller signal due to superconducting-shielding current. The system allows us to investigate both ferromagnetic and superconducting properties in a single experiment. Since one of the uranium compounds, $\mathrm{UGe}_{2}$ becomes the ferromagnetic superconductor only in high pressure, the system was installed to a piston-cylinder-type pressure cell.
\end{abstract}

Keywords: uranium compounds; magnetic measurements; ferromagnetism; superconductivity; high pressure equipment

\section{Introduction}

Coexistence of ferromagnetism and superconductivity is one of the fascinating properties of metallic uranium compounds. Since discovery of a phase in which ferromagnetism and superconductivity coexist [1], uranium digermanide $\mathrm{UGe}_{2}$ has been extensively investigated ([2] and reference therein). The U $5 f$-electrons are considered to be responsible for both the properties. One of the main issues in the research of the ferromagnetic superconductor is to reveal mechanism how the superconductivity can survive in the presence of strong internal field (exchange field) caused by the ferromagnetism. Theoretically, possible realization of spin-triplet nonunitary superconducting order parameter was proposed, because it is not affected by the exchange field [3-6]. In addition, with related to this issue, influence of ferromagnetic domain walls has been also discussed in many articles [3-6,7,8]. Fomin has mentioned in the article [4], if the nonunitary superconducting state was realized, the domain walls should work as the weak links for the superconductivity. On the contrary, the opposite prediction has been proposed in several papers [7,8]. According to the Ginzberg-Landau theory, superconducting transition temperature can be enhanced near the domain walls, which means superconductivity is enhanced at the

*Corresponding author. Email: yamagu@sci.u-hyogo.ac.jp domain boundary. This behavior can be attributed to the electromagnetic effect, which is analogous to the surface superconductivity of ordinary superconductors.

At present experimental verification was not carried out and the situation is controversial. Therefore, in order to clarify this, we developed a new experimental set-up, where we can carry out simultaneous measurement on $\mathrm{dc}$ and ac magnetic properties in the ferromagnetic and superconducting phase. By using two types of magnetic sensors, we would investigate relation between the ferromagnetic domain structure and superconducting properties. In this report, the details of apparatus are presented and preliminary results are shown for the $\mathrm{UGe}_{2}$ crystals.

\section{Sample}

$\mathrm{UGe}_{2}$ that was used in this research, was prepared in Laboratory of Alpha-Ray Emitters, Institute for Materials Research, Tohoku University. First, stoichiometric amounts of natural uranium and germanium of high purity were melted to form polycrystalline sample. Then single crystals were grown by the Czochralski pulling method using a tetra-arc furnace in a pure argon atmosphere. Figure 1 shows temperature dependence of electrical resistivity between 2 and $250 \mathrm{~K}$, at ambient pressure. The kink of resistivity indicates a ferromagnetic transition with the Curie temperature $T_{\text {Curie }}=52 \mathrm{~K}$, which was in good agreement 
with the previous results [1,9]. The residual ratio of resistivity is 180 . Two crystals were used for a check of the system, whose weights are 9.1 and $12.1 \mathrm{mg}$, respectively (denoted as sample A and sample B).

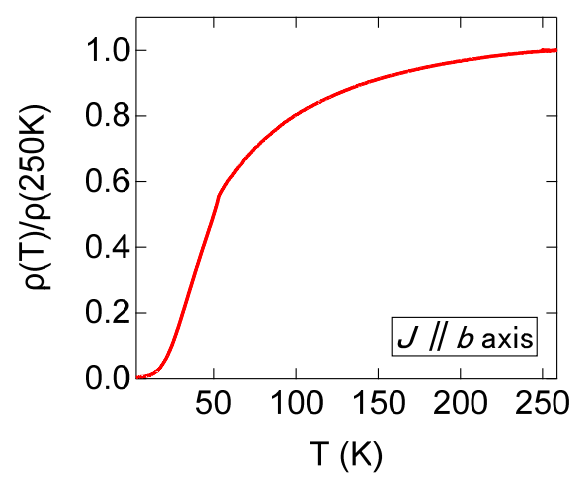

Figure 1. Temperature dependence of electrical resistivity of $\mathrm{UGe}_{2}$. The data are normalized by the value at $250 \mathrm{~K}$.

\section{Development}

\subsection{Overview}

We show in Figure 2 a cross-sectional view of the low-temperature part of the system. The system has been designed to fit the piston-cylinder-type high-pressure cell made of $\mathrm{NiCrAl}$ and $\mathrm{BeCu}$ [10]. The highest pressure of the equipment is about $2 \mathrm{GPa}$. The size of sample space is $8 \mathrm{~mm}$ in diameter, and $18 \mathrm{~mm}$ in height at around $1.2 \mathrm{GPa}$. Two types of magnetic sensor were employed in the system. The Hall-device sensor was directly installed inside of the pressure cell. On the other hand, a SQUID sensor was settled at the place far from the cell, because it have to be immersed in liquid helium.

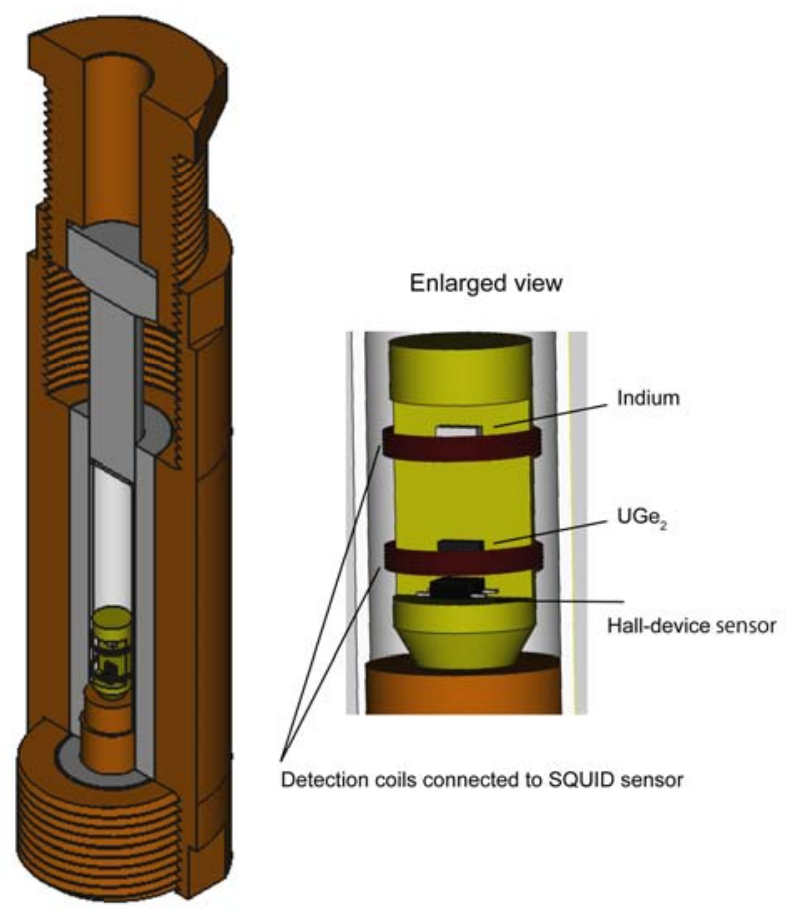

Figure 2. A cross-sectional view of a low-temperature part of the system.
Low-temperature experiments were carried out by using a ${ }^{3} \mathrm{He}-{ }^{4} \mathrm{He}$ dilution refrigerator (Cryoconcept model-200) down to $80 \mathrm{mK}$. In the experiment, the cell and SQUID sensor were settled at a mixing chamber plate and $4 \mathrm{~K}$-top flange, respectively. The connection between the cell and the SQUID sensor was made by a superconducting flux transformer, as is described in detail later.

The Daphne oil 7373 was used as pressure transmitting medium. A piece of pure indium $(99.9999 \%)$ was installed as a pressure manometer. Its superconducting transition temperature was used for pressure calibration. The same indium was also used for determining the absolute value of ac susceptibility. The dc magnetic field was applied by an 8 T superconducting magnet (Oxford Instruments Inc.)

\subsection{Hall-device magnetometry}

The magnetometer using the Hall device was made by referring to several papers $[11,12]$. The Hall device sensor used here is a commercial product of Asahi Kasei Microdevice Corp., model HG-106A GaAs Hall sensor. We tested several kinds of Hall devices, and the HG-106A provided the best performance among them. Hall voltage is $85 \mathrm{mV}$ at $50 \mathrm{mT}$ with applied current 10 $\mathrm{mA}$. The characteristic of the device is summarized in Table. 1 for $T=295$ and $4.2 \mathrm{~K}$ at ambient pressure. Applied pressure dependence of Hall voltage was found to be small at least up to $1.3 \mathrm{GPa}$. The size of the Hall device is $1.5 \times 2.5 \times 0.6 \mathrm{~mm}^{3}$, which is much smaller than the diameter of the sample space. In the experiment the sensor was located near the sample as close as possible in order to detect magnetic field produced by the sample magnetization. There are known parallel and perpendicular arrangements of the detection surface of the Hall sensor with respect to the applied field. In the parallel arrangement, there is an advantage that can reduce the background signal from applied fields. However, we adopted the perpendicular arrangement in this development because it has another advantage that enable us to calibrate the Hall sensor itself by applying external fields. In the case of perpendicular arrangement, the sensor detect total magnetic field due to the sample and applied field. By subtracting contribution from the applied field, we can deduce the field from the sample. The field is assumed to be proportional to the magnetization of the sample.

Table 1. Characteristic of HG-106A [13].

\begin{tabular}{lcc}
\hline Parameters & $T=295 \mathrm{~K}$ & $T=4.2 \mathrm{~K}$ \\
\hline Hall Voltage $(\mathrm{mV})$ & $85(50 \mathrm{mT}, 10 \mathrm{~mA})$ & 97.05 \\
Input Resistance $(\Omega)$ & 600 & 97.79 \\
Output Resistance $(\Omega)$ & 1500 & 2700 \\
Offset Voltage $(\mathrm{mV})$ & $<16$ & $\sim 14$ \\
\hline
\end{tabular}

\subsection{Magnetometry by SQUID sensor}

Figure 3 shows a schematic diagram of the SQUID magnetometer. The SQUID sensor is a product of 
TRISTAN technology, LT-SQUID. As described above, the sensor was located far from the sample. In the case of our dilution refrigerator, the distance between the pressure cell and the SQUID sensor was about $50 \mathrm{~cm}$. The connection between them was made by niobium-titanium superconducting wire $(0.15 \mathrm{~mm}$ in diameter) with a superconducting lead-tin alloy shield (flux transformer). In general the SQUID sensor is so sensitive that it is easily disturbed by noise due to fluctuation of applied magnetic field. Therefore, a parallel inductance and small resistance were inserted at input terminals to adjust the sensitivity and to reduce the noise.

SQUID sensor is used to detect ac field response from the samples. Since the detection coils were located inside of the pressure cell, the amount of the background signal from the cell was negligible. The ac field was applied by a home-made small superconducting solenoid coil. The coil constant was $11 \mathrm{mT} / \mathrm{A}$. The coil was wound around the high pressure cell. To reduce the shielding effect from the $\mathrm{BeCu}$ body of the cell, the frequency of applied ac field was kept below $15 \mathrm{~Hz}$.

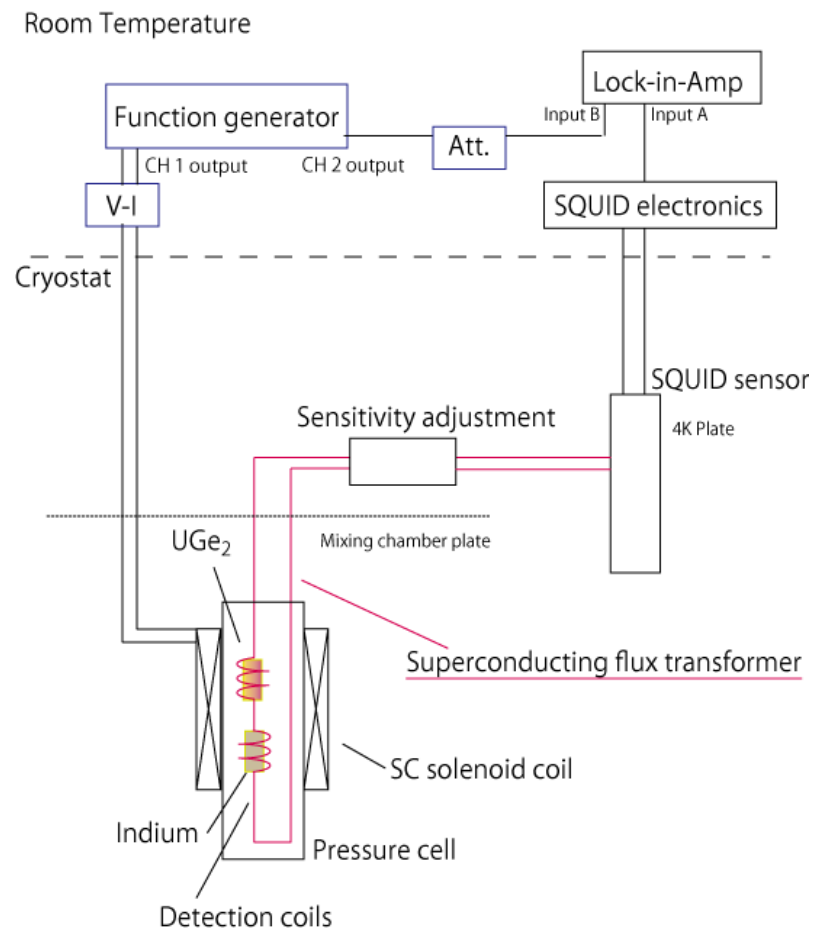

Figure 3. Schematic diagram of SQUID magnetometer.

\section{Results}

Figure 4 shows results in the measurement of the magnetization process of $\mathrm{UGe}_{2}$ around $1.1 \mathrm{GPa}$ and 750 $\mathrm{mK}$. The value of magnetic field measured by Hall sensor is about $10 \mathrm{mT}$ and $8 \mathrm{mT}$ at $0.1 \mathrm{~T}$, for the sample $\mathrm{A}$ and $\mathrm{B}$, respectively, at which, the magnetization of $\mathrm{UGe}_{2}$ is considered to be provisionally saturated [14]. The values of the detected field are almost consistent with a simulation that takes into consideration the arrangement of the sample and sensor and the $\mathrm{UGe}_{2}$ with saturation magnetization. According to the simulation, the strength of the field that Hall sensor detect is quite sensitive with the arrangement. For example, in the case of the arrangement of the sample B, a little change of the sensor position of only $0.7 \mathrm{~mm}$ lead to a large change of the field from 8 to $12 \mathrm{mT}$. The observed value of $8 \mathrm{mT}$ is within this expectation.
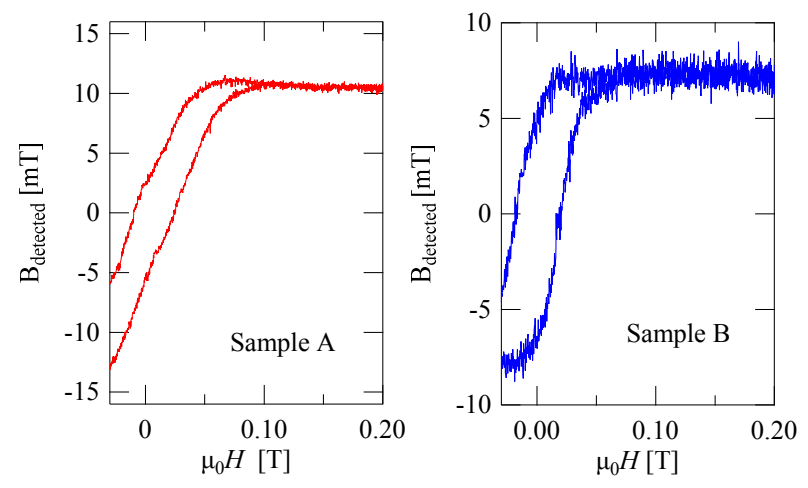

Figure 4. The magnetic field $\left(B_{\text {detected }}\right.$ ) produced by the samples as a function of the applied field at $750 \mathrm{mK}$. The value of $B_{\text {detected }}$ depends on the arrangement of sample and Hall sensor, and be proportional to magnetization of the sample.

Finally, we show the results of measurement by the SQUID sensor in Figure 5 for the sample A and B. The frequency of the applied ac field was $11 \mathrm{~Hz}$. Both the data shows a clear indication of superconducting transition where a rapid drop of ac susceptibility is observed. The transition temperatures was $0.6 \mathrm{~K}$ for the sample $\mathrm{A}$ at $1.03 \mathrm{GPa}$ and $0.65 \mathrm{~K}$ for the sample $\mathrm{B}$ at $1.15 \mathrm{GPa}$, respectively. These values are quite consistent with the reported phase diagram [15].

In the data of ac susceptibility measurements, no influence was observed from the Hall sensor located near the sample. This might be because the Hall sensor is settled at the outside of the detection coil. We can neglect parasitic contribution due to the Hall sensor through the experiment.
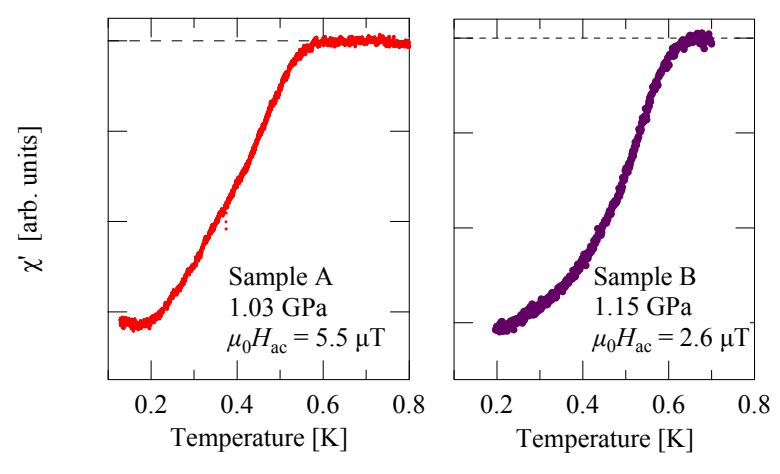

Figure 5. Ac susceptibility measured by the SQUID sensor. 


\section{Conclusion}

The magnetic-measurement system was developed for investigation of the ferromagnetic superconducting phase in $\mathrm{UGe}_{2}$. The $\mathrm{dc}$ magnetization and ac susceptibility well reproduced the previous results. The further experiment is now in progress to reveal the relation between magnetic domain structure and superconducting properties in the ferromagnetic superconducting phase.

\section{Acknowledgements}

The authors wish to thank H. Mizushima for the assistance of electrical resistivity measurements. This work was supported by JSPS KAKENHI (No. $16 \mathrm{~K} 05450$ ), and carried out in the joint research in Institute for Materials Research, Tohoku University.

\section{References}

[1] S.S. Saxena, P. Agarwal, K. Ahilan, F.M. Grosche, R.K.W. Haselwimmer, M.J. Steiner, E. Pugh, I.R. Walker, S.R. Julian, P. Monthoux, G.G. Lonzarich, A. Huxley, I. Sheikin, D. Braithwaite and J. Flouquet, Superconductivity on the border of itinerant-electron ferromagnetism in $\mathrm{UGe}_{2}$, Nature 406 (2000), pp. 587-592.

[2] D. Aoki and J. Flouquet, Ferromagnetism and superconductivity in uranium compounds, J. Phys. Soc. Jpn. 81 (2012), pp. 011003-1-11.

[3] K. Machida and T. Ohmi, Phenomenological theory of ferromagnetic superconductivity, Phys. Rev. Lett. 86 (2001), pp. 850-853.

[4] I.A. Fomin, Symmetry of the order parameter in the $\mathrm{UGe}_{2}$ superconductor, JETP Lett. 74 (2001), pp. 111-114.

[5] V.P. Mineev, Superconducting states in ferromagnetic metals, Phys. Rev. B 66 (2002), pp. 134504-1-7.
[6] I.A. Fomin, Gap nodes in the superconducting phase of the itinerant ferromagnet $\mathrm{UGe}_{2}$, arXiv: cond-mat. (2002), pp. 0207152v1-1-7.

[7] A.I. Buzdin and A.S. Mel'nikov, Domain wall superconductivity in ferromagnetic superconductors, Phys. Rev. B 67 (2003), pp. 020503(R)-1-4.

[8] K.V. Samokhin and D. Shirokoff, Phenomenological theory of superconductivity near domain walls in ferromagnets, Phys. Rev. B 71 (2005), pp. 104527-1-6.

[9] Y. Ōnuki, I. Ukon, S.W. Yun, I. Umehara, K. Satoh, T. Fukuhara, H. Sato, S. Takayanagi, M. Shikama and A. Ochiai, Magnetic and electrical properties of U-Ge intermetallic compounds, $J$. Phys. Soc. Jpn. 61 (1992), pp. 293-299.

[10] A commercial product of C\&T Factory co., Ltd.

[11] T. Nishioka and N.K. Sato, New type of magnetization equipment using a commercial Hall sensor, J. Mag. Mag. Mater. 272-276 (2004) pp. 2305-2306.

[12] M. Mizuo, Y. Kawamura, T. Nishioka, H. Kato and M. Matsumura, Performance of magnetometer using a commercial Hall sensor, J. Phys.: Conf. Ser. 200 (2010) pp. 112007-1-4.

[13] Asahi Kasei Microdevice Corp., Datasheet of HG-106A, 2013.

[14] T. Sakon, S. Saito, K. Koyama, S. Awaji, I. Sato, T. Nojima, K. Watanabe and N.K. Sato, Experimental investigation of giant magnetocrystalline anisotropy of $\mathrm{UGe}_{2}$, Phys. Scr. 75 (2007) pp. 546-550.

[15] A. Huxley, I. Sheikin, E. Ressouche, N. Kernavanois, D. Braithwaite, R. Calemczuk and J. Flouquet, $\mathrm{UGe}_{2}$ : A ferromagnetic spin-triplet superconductor, Phys. Rev. B 63 (2001) pp. 144519-1-13. 